\title{
Nanomedicine: Future Promising Therapy on Treating Meibomian Gland Dysfunction
}

\section{Liangbo Chen ${ }^{1,2}$, Yao Fu ${ }^{1,2 *}$ and Yan Pang1,2*}

${ }^{1}$ Department of Ophthalmology, Ninth People's Hospital, Shanghai JiaoTong University School of Medicine, Shanghai, China

${ }^{2}$ Shanghai Key Laboratory of Orbital Diseases and Ocular Oncology, Shanghai, China

*Corresponding authors: Yao Fu, Yan Pang, Shanghai Key Laboratory of Orbital Diseases and Ocular Oncology, Shanghai, China, Email: drfuyao@126.com, py-1985@163.com

\section{Perspective Article}

Volume 4 Issue 3

Received Date: December 10, 2019

Published Date: December 16, 2019

DOI: $10.23880 /$ nnoa-16000170

\begin{abstract}
Meibomian gland dysfunction (MGD) is a chronic inflammation-related disease, which can cause instability of tear film, discomfort of the ocular surface and eyelids, and finally lead to dry eye disease (DED). The current clinical therapy has the following disadvantages, such as time-consuming, general treatment effect, and expensive treatment. Nanomedicine has been used in the treatment of some ocular surface diseases. For example, nanoparticles have been used in some eye drops formulations as well as eye patches, which can enhance the water solubility of the drugs, prolong the action time, and reduce the toxicity. In this review, we have introduced MGD and its' current clinical therapy. Since nanomedicine has already participated in treating DED patients and got satisfactory outcome, we believe that it will also have promising prospects in treating MGD.
\end{abstract}

Keywords: Inflammatory; Nanomedicine; Meibomian Gland Dysfunction

Abbreviations: Mgd: Meibomian Gland Dysfunction; Ipl: Intense Pulsed Light; Tgf-B1: Transforming Growth Factor Beta-1; Tcsa: Topical Cyclosporine A; Nps: Nanoparticles; Nitbut: Noninvasive Tear Break-Up Time; TMH: Tear Meniscus Height.

\section{Introduction}

The meibomian gland is a type of sebaceous gland with tubuloacinar structure and holocrine function, located in the superior and inferior tarsal plates [1]. Meibomian glands secrete meibum, a compound made up of polar lipids (phospholipids) and nonpolar lipids (cholesterol, wax esters, cholesterol esters) [2]. Meibum forms the lipid layer at the surface of the tear film, which prevents dry eye by reducing ocular surface water evaporation and collapse of the tear film [3]. In 2011, meibomian gland dysfunction (MGD) was defined as a chronic, diffuse abnormality of the meibomian glands, commonly characterized by terminal duct obstruction and/or qualitative/quantitative changes in the glandular secretion by the International Workshop [1]. Furthermore, MGD can lead to altered tear film composition, ocular surface disease, ocular and eyelid discomfort, and evaporative dry eye [4]. According to a report published in 2011, nearly $50.0 \%-62.5 \%$ of patients with DED were diagnosed with MGD at the same 


\section{Nanomedicine \& Nanotechnology Open Access}

time [1]. Meanwhile, more and more patients are diagnosed with MGD year after year.

\section{Current Clinical Therapy}

The goal of treatment for MGD is to improve the flow of meibum and ultimately help restore tear film stability [5]. Here, commonly used clinical treatments of MGD are introduced.

\section{Lid Hygiene and Warm Compression}

Eyelid warming and massage are the basic treatments for MGD. In order to improve the basic treatment effect, an automatic machine was invented. Lipiflow (TearScience, inc.) was recently introduced as an automated way to warm and massage the eyelids. In three randomized clinical trials [6-8], application of Lipiflow alone could improve meibomian gland secretion, ocular symptoms, and the tear breakup time up to 1 or 3 months after treatment. In summary, the outcome was better than or at least similar to those who suffered daily conventional warm compression.

\section{Intense Pulsed Light (IPL)}

IPL therapy has already been an effective treatment for a series of dermatologic diseases, which results in the alleviation of telangiectasia and facial erythema [9]. Meanwhile, IPL therapy has been evaluated as a promising treatment for MGD. In two prospective, noncomparative studies [10,11], serial IPL therapy sessions obviously improved ocular symptoms and signs, including the meibomian gland secretion quality and quantity. Mechanisms proposed for IPL to improve MGD signs and symptoms include thrombosis of the perivascular system of the meibomian gland, heat transfer, and reduction of inflammatory cytokines in tears $[9,12]$. No doubt, further investigation should be carried out to clarify the mechanisms.

\section{Anti-Inflammation}

Comparing patients with and without MGD, the expression of proinflammatory mediators (interleukin $[\mathrm{IL}]-1 \beta$, IL-8, and matrix metalloproteinase 9) and transforming growth factor beta-1 (TGF-b1) was higher and lower, respectively [13]. Therefore, antiinflammatory treatment is important for MGD. Zhang, et al. [14] used 1\% azithromycin eye drops in 16 patients with MGD for 4 weeks and examined changes in the expression of anti-inflammatory and proinflammatory mediators in the conjunctiva and eyelid margin. It was found that topical application of azithromycin could reduce the inflammation in the ocular surface and eyelid of patients with MGD by altering the expression of proand anti-inflammatory mediators. Perry, et al. [15] investigated the efficacy of topical cyclosporine A $0.05 \%$ (tCsA) for the treatment of MGD in a prospective study. In the tCsA group, a significant improvement of fluorescein staining scores, tarsal telangiectasis, and lid margin vascular injections were found compared to the control group. It suggested that topical CsA may be helpful in the treatment of MGD.

\section{Vitamin D3}

Vitamin D3 is also a promising drug for MGD. Since hyperkeratinization is a major cause of obstructive MGD, maxacalcitol (a noncalcemic analogue of the active form of vitamin D3) is expected to be effective in patients with MGD, which is proved to be effective for psoriasis and ichthyosis. Arita, et al. [16] applied maxacalcitol ointment to the lid margins of patients with obstructive MGD for 2 months. A significant improvement in clinical parameters (the tear breakup time, scores for plugging of meibomian gland orifices and lid margin vascularity and the meibum grade) was observed.

In general, the current clinical therapy of MGD mainly includes physical therapy and drug treatment. However, all the above-mentioned treatments are suffering from long time course, ineffective treatment, and high medical expense. More and more scientists currently focusing on the field of MGD treatment, hoping to develop more effective and convenient therapy.

\section{Nanomedicine in Ocular Surface Disease}

Topical eyedrops have poor ocular bioavailability owing to the rapid tear film turnover, corneal barrier, and quick tear drainage [17]. Nanomedicine can increase solubility of hydrophobic drug in aqueous solution, sustained drug release with improved efficacy and reduced toxicity, enhance drug penetration through ocular barriers and extend drug retention time, and even some nanomedicines can target specific cells and tissues. Therefore, a variety of nanomedicines have been developed for ocular drug delivery, such as polymeric nanoparticles (NPs), liposomes, dendrimers and micelles [18].

CsA mentioned above suffered a lot to develop aqueous eyedrops due to hydrophobic nature (solubility $10 \mathrm{mg} / \mathrm{ml}$ ) and large molecular weight (1202.6 Da). 


\section{Nanomedicine \& Nanotechnology Open Access}

Cequa@ (OTX-101) is a micelle formulation containing $0.09 \%$ CsA. The particle size of micelle is $12-20 \mathrm{~nm}$. The micelle formulation is composed of polyoxyl hydrogenated castor oil (HCO-40) and octoxynol-40 (OC40 ), which could simultaneously form thermodynamic stable micelles through hydrogen-bonding. On August 14th, 2018, Cequa@ was approved by US FDA, twice daily for the treatment of signs and symptoms of DED in adult patients [19]. Gold nanoparticles, palladium (Pd) shell and gelatin had been applicated to fabricate GNRs @ Pd hydrogel eye patch by genipin cross-linking [20]. After being attached to the lacrimal gland, the hydrogel patch could convert various rays of light into heat, stimulated the lacrimal gland to produce more tears and relieve the dry eye. After application in DED patients, their measurements of the first noninvasive tear break-up time (NITBUT), the average NITBUT, red eye analysis, combined with the tear meniscus height (TMH) all improved compared to the control group.

\section{Summary and Future Perspective}

This review simply described a world wild ocular surface disease that affects people's quality of life, namely MGD, which is also one of the important causes of DED. However, for MGD, a chronic disease, the treatment is usually a long course. For example, when eye drops are needed for treatment, it usually takes 1-3 months, and more than 3 times a day. And for physical therapy such as heating and eyelid massage, patients usually will be treated at intervals, with varying length which depends on the severity. Long-term and high-frequency therapy will lead to huge financial burden and patients' nonadherence, which will dramatically affect the treatment effect. By combining nanotechnology with traditional therapy, long-term sustained release of eye drops as well as drug loading microneedle patches will be promising therapies to treat MGD in the future.

\section{Acknowledgments}

This work was supported by Shanghai Municipal Education Commission-Gaofeng Clinical Medicine Grant Support (20191820) and The Science and Technology Commission of Shanghai (17DZ2260100).

\section{References}

1. Nichols KK, Foulks GN, Bron AJ, Glasgow BJ, Dogru M, et al. (2011) The international workshop on meibomian gland dysfunction: executive summary. Invest Ophthalmol Vis Sci 52(4): 1922-1929.
2. Foulks GN, Bron AJ (2003) Meibomian gland dysfunction: a clinical scheme for description, diagnosis, classification, and grading. Ocul Surf 1(3): 107-126.

3. Altin Ekin M, Karadeniz Ugurlu S, Kahraman HG (2019) Meibomian Gland Dysfunction and Its Association With Ocular Discomfort in Patients With Ocular Prosthesis. Eye Contact Lens.

4. Chhadva p, Goldhardt R, Galor A (2017) Meibomian Gland Disease: The Role of Gland Dysfunction in Dry Eye Disease. Ophthalmology 124(11S): S20-S26.

5. Qiao J, Yan X (2013) Emerging treatment options for meibomian gland dysfunction. Clin Ophthalmol 7: 1797-1803.

6. Lane SS, DuBiner HB, Epstein RJ, Ernest PH, Greiner JV, et al. (2012) A new system, the LipiFlow, for the treatment of meibomian gland dysfunction. Cornea 31(4): 396-404.

7. Baumann A, Cochener B (2014) Meibomian gland dysfunction: a comparative study of modern treatments. J Fr Ophtalmol 37(4): 303-312.

8. Finis D, Hayajneh J, Konig C, Borrelli M, Schrader S (2014) Evaluation of an automated thermodynamic treatment (LipiFlow(R)) system for meibomian gland dysfunction: a prospective, randomized, observermasked trial. Ocul Surf 12(2): 146-154.

9. Craig JP, Chen YH, Turnbull PR (2015) Prospective trial of intense pulsed light for the treatment of meibomian gland dysfunction. Invest Ophthalmol Vis Sci 56(3): 1965-1970.

10. Dell SJ, Gaster RN, Barbarino SC, Cunningham DN (2017) Prospective evaluation of intense pulsed light and meibomian gland expression efficacy on relieving signs and symptoms of dry eye disease due to meibomian gland dysfunction. Clin Ophthalmol 11: 817-827.

11. Albietz JM, Schmid KL (2018) Intense pulsed light treatment and meibomian gland expression for moderate to advanced meibomian gland dysfunction. Clin Exp Optom 101(1): 23-33.

12. Liu R, Rong B, Tu P, Tang Y, Song W (2017) Analysis of Cytokine Levels in Tears and Clinical Correlations After Intense Pulsed Light Treating Meibomian Gland Dysfunction. Am J Ophthalmol 183: 81-90. 


\section{Nanomedicine \& Nanotechnology Open Access}

13. Amano S (2018) Meibomian Gland Dysfunction: Recent Progress Worldwide and in Japan. Invest Ophthalmol Vis Sci 59(14): DES87-DES93.

14. Zhang L, Su Z, Zhang Z, Lin J, Li DQ (2015) Effects of azithromycin on gene expression profiles of proinflammatory and anti-inflammatory mediators in the eyelid margin and conjunctiva of patients with meibomian gland disease. JAMA Ophthalmol 133(10): 1117-1123.

15. Perry HD, Doshi-Carnevale S, Donnenfeld ED, Solomon R, Biser SA, et al. (2006) Efficacy of commercially available topical cyclosporine A $0.05 \%$ in the treatment of meibomian gland dysfunction. Cornea 25(2): 171-175.

16. Arita R, Kawashima M, Ito M, Tsubota K (2017) Clinical safety and efficacy of vitamin D3 analog ointment for treatment of obstructive meibomian gland dysfunction. BMC Ophthalmol 17(1): 84.
17. Meng T, Kulkarni V, Simmers R, Brar V, Xu Q (2019) Therapeutic implications of nanomedicine for ocular drug delivery. Drug Discov Today 24(8): 1524-1538.

18. Weng Y, Liu J, Jin S, Guo W, Liang X, et al. (2017) Nanotechnology-based strategies for treatment of ocular disease. Acta Pharm Sin B 7(3): 281-291.

19. Mandal A, Gote V, Pal D, Ogundele A, Mitra AK (2019) Ocular Pharmacokinetics of a Topical Ophthalmic Nanomicellar Solution of Cyclosporine (Cequa(R)) for Dry Eye Disease. Pharm Res 36(2): 36.

20. Pang Y, Wei C, Li R, Wu Y, Liu W, et al. (2019) Photothermal conversion hydrogel based mini-eye patch for relieving dry eye with long-term use of the light-emitting screen. Int J Nanomedicine 14: 51255133. 